\title{
Theoretical Error Analysis of Solution for Two-Dimensional Stochastic Volterra Integral Equations by Haar Wavelet
}

\author{
M. Fallahpour ${ }^{1} \cdot$ M. Khodabin $^{1}$ (D) K. Maleknejad ${ }^{1}$
}

Published online: 29 October 2019

(c) The Author(s) 2019

\begin{abstract}
The finding an efficient way to the approximate solutions of the stochastic integral equations is an essential requirement. In this paper we discuss the convergence analysis of the two-dimensional Haar wavelet functions (2D-HWFs) method for solve 2D linear stochastic Volterra integral equation. The illustrative examples are included to demonstrate the validity and applicability of this numerical method.
\end{abstract}

Keywords Haar wavelet function - Two-dimensional integral equation · Stochastic integral equation · Volterra integral equation · Brownian motion process $\cdot$ Ito integral

Mathematics Subject Classification $65 \mathrm{C} 20 \cdot 60 \mathrm{H} 20 \cdot 45 \mathrm{Axx} \cdot 45 \mathrm{Dxx} \cdot 65 \mathrm{~T} 60$

\section{Introduction}

In very of the engineering problems, we see the important role of 2D integral equations [1,2], where are produced from a differential equation. Also if we import the statistical noise into a general hyperbolic differential equation we can obtain 2D linear stochastic Volterra integral equation of the second kind, i.e.

$$
\begin{aligned}
g(x, y)= & f(x, y)+\int_{0}^{y} \int_{0}^{x} K_{1}(x, y, s, t) g(s, t) d s d t \\
& +\int_{0}^{y} \int_{0}^{x} K_{2}(x, y, s, t) g(s, t) d B(s) d B(t) \\
& (x, y) \in[0,1] \times[0,1], \quad s \leqslant x<t \leqslant y .
\end{aligned}
$$

M. Khodabin

m-khodabin@kiau.ac.ir

M. Fallahpour

M.Fallahpour@kiau.ac.ir

K. Maleknejad

maleknejad@iust.ac.ir

1 Department of Mathematics, Karaj Branch, Islamic Azad University, Karaj, Iran 
where $B(t)$ is Brownian motion process. In the last years, the numerous numerical methods have been introduced to estimate the solution of 2D ordinary integral equations [3-8] and many other papers, whereas it is worked few for 2D stochastic integral equations. Recently, some authors have proposed the methods to solve 2D stochastic integral equations [9-14]. Especially, Fallahpour et al. [9], included HWFs method to solve such equations without the error analysis. In this paper, we use HWFs method with the error and convergence analysis to derive approximate solution of (1).

This paper is organized as follows:

In next section we present HWFs. In section "Haar Wavelets Numerical Method", the method is applied to solve Eq. (1). The error analysis of this method is discussed in section "Error Analysis". Section "Numerical Example", uses some numerical examples to show the convergence of the proposed method and compares it with the block-pulse functions (BPFs) method as proposed in $[10,11]$. Finally, conclusion is given, in section "Conclusion".

\section{Haar Wavelets}

As we know, for HWFs we can write

$$
\psi_{j, i}(z)=2^{j / 2} \psi\left(2^{j} z-i\right),
$$

so on the interval $[0,1)$ we have

$$
h_{1}(z)=\left\{\begin{array}{l}
1, \text { for } z \in[0,1) \\
0, \text { otherwise }
\end{array}\right.
$$

and

$$
h_{i}(z)=\left\{\begin{array}{cc}
1, & \text { for } z \in[\alpha, \beta) \\
-1, & \text { for } z \in[\beta, \gamma) \\
0, & \text { otherwise, }
\end{array}\right.
$$

for $i=2,3, \ldots$, where

$$
\alpha=\frac{n}{m}, \quad \beta=\frac{(n+0.5)}{m}, \quad \gamma=\frac{(n+1)}{m},
$$

so

$$
m=2^{\ell}, \ell=0,1, \ldots, n=0,1, \ldots, m-1 .
$$

The integer $\ell$ and $n$, are the wavelet level and the translation parameter, respectively. Also Haar wavelets $h_{i}(z)$ are pairwise orthonormal in the interval $[0,1)$ as

$$
\int_{0}^{1} h_{i}(z) h_{j}(z) d y=\delta_{i j},
$$

where $\delta_{i j}$ is Kronecker delta. We display the maximum value of $\ell$ by $J$ where $M=2^{J}$. Therefore for any square integrable function $f(z)$ we have

$$
f(z) \approx \sum_{i=1}^{2 M} a_{i} h_{i}(z) .
$$


We show the ordinary Volterra ihtegral of Haar wavelet as

$$
p_{i}(z)=\int_{0}^{z} h_{i}(u) d u
$$

where by using HWFs we get

$$
p_{i}(z)=\left\{\begin{array}{cl}
z-\alpha, & \text { for } z \in[\alpha, \beta) \\
\gamma-z, & \text { for } z \in[\beta, \gamma) \\
0, & \text { elsewhere. }
\end{array}\right.
$$

Also for the stochastic Volterra integral we can introduce

$$
q_{i}(z)=\int_{0}^{z} h_{i}(u) d B(u),
$$

where similarly by Haar wavelet definition we have

$$
q_{i}(z)= \begin{cases}B(z)-B(\alpha), & \text { for } z \in[\alpha, \beta) \\ 2 B(\beta)-B(\alpha)-B(z), & \text { for } z \in[\beta, \gamma) \\ 0, & \text { elsewhere }\end{cases}
$$

\section{Haar Wavelets Numerical Method}

In this section, we solve Eq. (1) by using 2D-HWFs. For Haar wavelet approximation of a function $f(x, y)$, we use the collocation points

$$
\begin{gathered}
x_{m}=\frac{m-0.5}{2 M}, m=1,2, \ldots, 2 M, \\
y_{n}=\frac{n-0.5}{2 N}, n=1,2, \ldots, 2 N .
\end{gathered}
$$

\section{D-HWFs System}

A real-valued function $E(x, y)$ can be approximated using 2D-HWFs as

$$
E(x, y) \approx \sum_{d=1}^{2 M} \sum_{e=1}^{2 N} b_{d, e} h_{d}(x) h_{e}(y)
$$

where the unknown coefficients $b_{d, e}$ 's, have calculated in [9]. Also we consider a function $E(x, y, s, t)$ where is approximated using $2 \mathrm{D}-\mathrm{HWF}$ as

$$
E(x, y, s, t) \approx \sum_{d=1}^{2 M} \sum_{e=1}^{2 N} b_{d, e}(x, y) h_{d}(s) h_{e}(t),
$$

so by substituting the collocation points

$$
s_{i}=\frac{i-0.5}{2 M}, i=1,2, \ldots, 2 M,
$$

and

$$
t_{j}=\frac{j-0.5}{2 N}, j=1,2, \ldots, 2 N,
$$


we get

$$
E\left(x, y, s_{i}, t_{j}\right) \approx \sum_{d=1}^{2 M} \sum_{e=1}^{2 N} b_{d, e}(x, y) h_{d}\left(s_{i}\right) h_{e}\left(t_{j}\right),
$$

where the solution of System (7) is calculated from Corollary (1) in [9].

\section{D-Linear Stochastic Volterra Integral Equation}

We approximate the functions $K_{1} g$ and $K_{2} g$ in (1) by using 2D-HWFs as

$$
K_{1}(x, y, s, t) g(s, t) \approx \sum_{i=1}^{2 M} \sum_{j=1}^{2 N} b_{i, j}(x, y) h_{i}(s) h_{j}(t)
$$

and

$$
K_{2}(x, y, s, t) g(s, t) \approx \sum_{i=1}^{2 M} \sum_{j=1}^{2 N} c_{i, j}(x, y) h_{i}(s) h_{j}(t),
$$

respectively. With these approximations, Eq. (1) can be writen as

$$
\begin{aligned}
g(x, y)= & f(x, y)+\int_{0}^{y} \int_{0}^{x} \sum_{i=1}^{2 M} \sum_{j=1}^{2 N} b_{i, j}(x, y) h_{i}(s) h_{j}(t) d s d t \\
& +\int_{0}^{y} \int_{0}^{x} \sum_{i=1}^{2 M} \sum_{j=1}^{2 N} c_{i, j}(x, y) h_{i}(s) h_{j}(t) d B(s) d B(t) .
\end{aligned}
$$

By applying Eqs. (3) and (4) in (8), we have

$$
g(x, y)=f(x, y)+\sum_{i=1}^{2 M} \sum_{j=1}^{2 N} b_{i, j}(x, y) p_{i}(x) p_{j}(y)+\sum_{i=1}^{2 M} \sum_{j=1}^{2 N} c_{i, j}(x, y) q_{i}(x) q_{j}(y) .
$$

The collocation points (5) and (6), give

$$
\begin{aligned}
g\left(x_{m}, y_{n}\right)= & f\left(x_{m}, y_{n}\right)+\sum_{i=1}^{2 M} \sum_{j=1}^{2 N} b_{i, j}\left(x_{m}, y_{n}\right) p_{i}\left(x_{m}\right) p_{j}\left(y_{n}\right) \\
& +\sum_{i=1}^{2 M} \sum_{j=1}^{2 N} c_{i, j}\left(x_{m}, y_{n}\right) q_{i}\left(x_{m}\right) q_{j}\left(y_{n}\right) .
\end{aligned}
$$

Now if we replace $b_{i, j}$ 's and $c_{i, j}$ 's by the given expressions of Corollary 1 in [9], we get

$$
\begin{aligned}
g\left(x_{m}, y_{n}\right)= & f\left(x_{m}, y_{n}\right)+\frac{p_{1}\left(x_{m}\right) p_{1}\left(y_{n}\right)}{2 M \times 2 N} \sum_{d=1}^{2 M} \sum_{e=1}^{2 N} K_{1}\left(x_{m}, y_{n}, s_{d}, t_{e}\right) g\left(s_{d}, t_{e}\right) \\
& +\sum_{i=2}^{2 M} \frac{p_{i}\left(x_{m}\right) p_{1}\left(y_{n}\right)}{\rho_{1} \times 2 N}\left(\sum_{d=\alpha_{1}}^{\beta_{1}} \sum_{e=1}^{2 N} K_{1}\left(x_{m}, y_{n}, s_{d}, t_{e}\right) g\left(s_{d}, t_{e}\right)\right. \\
& \left.-\sum_{d=\beta_{1}+1}^{\gamma_{1}} \sum_{e=1}^{2 N} K_{1}\left(x_{m}, y_{n}, s_{d}, t_{e}\right) g\left(s_{d}, t_{e}\right)\right)+\sum_{j=2}^{2 N} \frac{p_{1}\left(x_{m}\right) p_{j}\left(y_{n}\right)}{2 M \times \rho_{2}}
\end{aligned}
$$




$$
\begin{aligned}
& \times\left(\sum_{d=1}^{2 M} \sum_{e=\alpha_{2}}^{\beta_{2}} K_{1}\left(x_{m}, y_{n}, s_{d}, t_{e}\right) g\left(s_{d}, t_{e}\right)\right. \\
& \left.-\sum_{d=1}^{2 M} \sum_{e=\beta_{2}+1}^{\gamma_{2}} K_{1}\left(x_{m}, y_{n}, s_{d}, t_{e}\right) g\left(s_{d}, t_{e}\right)\right) \\
& +\sum_{i=2}^{2 M} \sum_{j=2}^{2 N} \frac{p_{i}\left(x_{m}\right) p_{j}\left(y_{n}\right)}{\rho_{1} \times \rho_{2}}\left(\sum_{d=\alpha_{1}}^{\beta_{1}} \sum_{e=\alpha_{2}}^{\beta_{2}} K_{1}\left(x_{m}, y_{n}, s_{d}, t_{e}\right) g\left(s_{d}, t_{e}\right)\right. \\
& -\sum_{d=\alpha_{1}}^{\beta_{1}} \sum_{e=\beta_{2}+1}^{\gamma_{2}} K_{1}\left(x_{m}, y_{n}, s_{d}, t_{e}\right) g\left(s_{d}, t_{e}\right)-\sum_{d=\beta_{1}+1}^{\gamma_{1}} \sum_{e=\alpha_{2}}^{\beta_{2}} K_{1}\left(x_{m}, y_{n}, s_{d}, t_{e}\right) g\left(s_{d}, t_{e}\right) \\
& \left.+\sum_{d=\beta_{1}+1}^{\gamma_{1}} \sum_{e=\beta_{2}+1}^{\gamma_{2}} K_{1}\left(x_{m}, y_{n}, s_{d}, t_{e}\right) g\left(s_{d}, t_{e}\right)\right)+\frac{q_{1}\left(x_{m}\right) q_{1}\left(y_{n}\right)}{2 M \times 2 N} \\
& \times \sum_{i=1}^{2 M} \sum_{j=1}^{2 N} K_{2}\left(x_{m}, y_{n}, s_{d}, t_{e}\right) g\left(s_{d}, t_{e}\right)+\sum_{i=2}^{2 M} \frac{q_{i}\left(x_{m}\right) q_{1}\left(y_{n}\right)}{\rho_{1} \times 2 N} \\
& \times\left(\sum_{d=\alpha_{1}}^{\beta_{1}} \sum_{e=1}^{2 N} K_{2}\left(x_{m}, y_{n}, s_{d}, t_{e}\right) g\left(s_{d}, t_{e}\right)-\sum_{d=\beta_{1}+1}^{\gamma_{1}} \sum_{e=1}^{2 N} K_{2}\left(x_{m}, y_{n}, s_{d}, t_{e}\right) g\left(s_{d}, t_{e}\right)\right) \\
& +\sum_{j=2}^{2 N} \frac{q_{1}\left(x_{m}\right) q_{j}\left(y_{n}\right)}{2 M \times \rho_{2}}\left(\sum_{d=1}^{2 M} \sum_{e=\alpha_{2}}^{\beta_{2}} K_{2}\left(x_{m}, y_{n}, s_{d}, t_{e}\right) g\left(s_{d}, t_{e}\right)\right. \\
& \left.-\sum_{d=1}^{2 M} \sum_{e=\beta_{2}+1}^{\gamma_{2}} K_{2}\left(x_{m}, y_{n}, s_{d}, t_{e}\right) g\left(s_{d}, t_{e}\right)\right)+\sum_{i=2}^{2 M} \sum_{j=2}^{2 N} \frac{q_{i}\left(x_{m}\right) q_{j}\left(y_{n}\right)}{\rho_{1} \times \rho_{2}} \\
& \times\left(\sum_{d=\alpha_{1}}^{\beta_{1}} \sum_{e=\alpha_{2}}^{\beta_{2}} K_{2}\left(x_{m}, y_{n}, s_{d}, t_{e}\right) g\left(s_{d}, t_{e}\right)-\sum_{d=\alpha_{1}}^{\beta_{1}} \sum_{e=\beta_{2}+1}^{\gamma_{2}} K_{2}\left(x_{m}, y_{n}, s_{d}, t_{e}\right)\right. \\
& \times g\left(s_{d}, t_{e}\right)-\sum_{d=\beta_{1}+1}^{\gamma_{1}} \sum_{e=\alpha_{2}}^{\beta_{2}} K_{2}\left(x_{m}, y_{n}, s_{d}, t_{d}\right) g\left(s_{d}, t_{e}\right) \\
& \left.+\sum_{d=\beta_{1}+1}^{\gamma_{1}} \sum_{e=\beta_{2}+1}^{\gamma_{2}} K_{2}\left(x_{m}, y_{n}, s_{d}, t_{e}\right) g\left(s_{d}, t_{e}\right)\right) \text {, }
\end{aligned}
$$

where $\alpha_{1}, \beta_{1}, \gamma_{1}, \rho_{1}$ and $\alpha_{2}, \beta_{2}, \gamma_{2}, \rho_{2}$ are defined in [9] and Eq. (9) shows a system $2 M \times 2 N$.

\section{Error Analysis}

In this section, we investigate the convergence and error analysis of HWFs method for solution (1). Here norm 2 is defined as

$$
\|f\|_{2}=[\underbrace{\int_{a}^{b} \int_{a}^{b} \ldots \int_{a}^{b}}_{m \text { times }}\left|f\left(x_{1}, x_{2}, \ldots, x_{m}\right)\right|^{2} d x_{1} d x_{2} \ldots d x_{m}]^{1 / 2} .
$$


To obtaining the typical convergence rate of HWFs method, we have:

Theorem 1 [15] For the differentiable function $p: D \subset R^{2} \longrightarrow R$, with

$$
\left\|p^{\prime}\right\| \leq M
$$

where $D$ and $M$ are a convex open set and a real number, respectively, we have

$$
|p(b)-p(a)| \leq M|b-a|
$$

for all $a, b \in D$.

Theorem 2 [16] Suppose that $f(s, t) \in L^{2}([0,1) \times[0,1))$ with bounded partial derivatives, $\left|\frac{\partial^{2} f}{\partial s \partial t}\right| \leq M$, and

$$
e_{m}(s, t)=f(s, t)-\sum_{i=0}^{m-1} \sum_{j=0}^{m-1} f_{i, j} h_{i}(s) h_{j}(t) .
$$

Then for $(s, t) \in D^{2}$, we have

$$
\left\|e_{m}\right\| \leq \frac{M}{3 m^{2}}
$$

Theorem 3 Consider $K(x, y, s, t) \in L^{2}([0,1) \times[0,1) \times[0,1) \times[0,1))$ with bounded partial derivatives,

$$
\left|\frac{\partial^{4} f}{\partial x \partial y \partial s \partial t}\right| \leq U
$$

and

$$
\hat{K}_{m}(x, y, s, t)=\sum_{a=0}^{m-1} \sum_{b=0}^{m-1} \sum_{c=0}^{m-1} \sum_{d=0}^{m-1} K_{a b c d} h_{a}(x) h_{b}(y) h_{c}(s) h_{d}(t),
$$

is $4 D$-HWF expansion of $K(x, y, s, t)$ where

$$
e_{a, b, c, d}(x, y, s, t)=K(x, y, s, t)-\hat{K}_{m}(x, y, s, t),
$$

then

$$
\left\|e_{a, b, c, d}\right\| \leq \frac{U}{9 m^{4}}
$$

for every $(x, y, s, t) \in D^{4}$.

Proof We can write

$$
\begin{aligned}
\left\|e_{a, b, c, d}\right\|^{2}= & \int_{0}^{1} \int_{0}^{1} \int_{0}^{1} \int_{0}^{1} \\
& \left(K(x, y, s, t)-\sum_{a=0}^{m-1} \sum_{b=0}^{m-1} \sum_{c=0}^{m-1} \sum_{d=0}^{m-1} K_{a b c d} h_{a}(x) h_{b}(y) h_{c}(s) h_{d}(t)\right)^{2} d t d s d y d x, \\
= & \int_{0}^{1} \int_{0}^{1} \int_{0}^{1} \int_{0}^{1}\left(\sum_{a=m}^{\infty} \sum_{b=m}^{\infty} \sum_{c=m}^{\infty} \sum_{d=m}^{\infty} K_{a b c d} h_{a}(x) h_{b}(y) h_{c}(s) h_{d}(t)\right)^{2} d t d s d y d x,
\end{aligned}
$$




$$
=\sum_{a=m}^{\infty} \sum_{b=m}^{\infty} \sum_{c=m}^{\infty} \sum_{d=m}^{\infty} \int_{0}^{1} \int_{0}^{1} \int_{0}^{1} \int_{0}^{1} K_{a b c d}^{2} h_{a}^{2}(x) h_{b}^{2}(y) h_{c}^{2}(s) h_{d}^{2}(t) d t d s d y d x
$$

where $a=2^{j_{1}}+k, b=2^{j_{2}}+k, c=2^{j_{3}}+k, d=2^{j_{4}}+k, m=2^{J}, J>0$ and

$$
K_{a b c d}=\int_{0}^{1} \int_{0}^{1} \int_{0}^{1} \int_{0}^{1} h_{a}(x) h_{b}(y) h_{c}(s) h_{d}(t) K(x, y, s, t) d t d s d y d x .
$$

Based on Haar wavelet definition, mean value theorem, Theorems 1 and 2 there are

$$
\eta_{j_{4}}, \alpha, \alpha^{\prime}, \eta_{j_{3}}, \beta, \beta^{\prime}, \eta_{j_{2}}, \gamma, \gamma^{\prime}, \eta_{j_{1}}, \theta, \theta^{\prime}
$$

where

$$
\begin{aligned}
& \alpha, \alpha^{\prime} \in\left[k 2^{-j_{4}},\left(k+\frac{1}{2}\right) 2^{-j_{4}}\right], \quad \beta, \beta^{\prime} \in\left[k 2^{-j_{3}},\left(k+\frac{1}{2}\right) 2^{-j_{3}}\right], \\
& \gamma, \gamma^{\prime} \in\left[k 2^{-j_{2}},\left(k+\frac{1}{2}\right) 2^{-j_{2}}\right], \theta, \theta^{\prime} \in\left[k 2^{-j_{1}},\left(k+\frac{1}{2}\right) 2^{-j_{1}}\right],
\end{aligned}
$$

such that

$$
\begin{aligned}
& K_{a b c d} \\
& =\int_{0}^{1} \int_{0}^{1} \int_{0}^{1} h_{a}(x) h_{b}(y) h_{c}(s)\left(\int_{0}^{1} h_{d}(t) K(x, y, s, t) d t\right) d s d y d x \\
& =\int_{0}^{1} \int_{0}^{1} \int_{0}^{1} h_{a}(x) h_{b}(y) h_{c}(s)\left(2^{\frac{-j_{4}}{2}-1}\left(\alpha-\alpha^{\prime}\right) \frac{\partial K\left(x, y, s, \eta_{j_{4}}\right)}{\partial t}\right) d s d y d x \\
& =\int_{0}^{1} \int_{0}^{1} h_{a}(x) h_{b}(y) \times 22^{\frac{-j_{4}}{2}-1}\left(\alpha-\alpha^{\prime}\right)\left(\int_{0}^{1} h_{c}(s) \frac{\partial K\left(x, y, s, \eta_{j_{4}}\right)}{\partial t} d s\right) d y d x \\
& =\int_{0}^{1} \int_{0}^{1} 2^{\frac{-j_{4}}{2}-\frac{-j_{3}}{2}-2} \times\left(\alpha-\alpha^{\prime}\right)\left(\beta-\beta^{\prime}\right)\left(\gamma-\gamma^{\prime}\right) \frac{\partial^{2} K\left(x, y, \eta_{j_{3}}, \eta_{j_{4}}\right)}{\partial s \partial t} h_{a}(x) h_{b}(y) d y d x .
\end{aligned}
$$

Finally we conclude

$$
\begin{aligned}
K_{a b c d}= & 2 \frac{-j_{4}}{2}-\frac{-j_{3}}{2}-\frac{-j_{2}}{2}-\frac{-j_{1}}{2}-4 \\
& \times\left(\alpha-\alpha^{\prime}\right)\left(\beta-\beta^{\prime}\right)\left(\gamma-\gamma^{\prime}\right)\left(\theta-\theta^{\prime}\right) \frac{\partial^{4} K\left(\eta_{j_{1}}, \eta_{j_{2}}, \eta_{j_{3}}, \eta_{j_{4}}\right)}{\partial x \partial y \partial s \partial t} .
\end{aligned}
$$

By using Eq. (2) and substituting Eq. (11) in (10) we get

$$
\begin{aligned}
\left\|e_{a, b, c, d}\right\|^{2}= & \sum_{a=m}^{\infty} \sum_{b=m}^{\infty} \sum_{c=m}^{\infty} \sum_{d=m}^{\infty} 2^{-j_{4}-j_{3}-j_{2}-j_{1}-8} \\
& \times\left(\alpha-\alpha^{\prime}\right)^{2}\left(\beta-\beta^{\prime}\right)^{2}\left(\gamma-\gamma^{\prime}\right)^{2}\left(\theta-\theta^{\prime}\right)^{2}\left|\frac{\partial^{4} K\left(\eta_{j_{1}}, \eta_{j_{2}}, \eta_{j_{3}}, \eta_{j_{4}}\right)}{\partial x \partial y \partial s \partial t}\right|^{2} \\
\leq & \sum_{a=m}^{\infty} \sum_{b=m}^{\infty} \sum_{c=m}^{\infty} \sum_{d=m}^{\infty} 2^{-j_{4}-j_{3}-j_{2}-j_{1}-8} \times 2^{-2 j_{1}} \times 2^{-2 j_{2}} \times 2^{-2 j_{3}} \times 2^{-2 j_{4}} \times U^{2}
\end{aligned}
$$




$$
\begin{aligned}
& =\sum_{a=m}^{\infty} \sum_{b=m}^{\infty} \sum_{c=m}^{\infty} \sum_{d=m}^{\infty} 2^{-3 j_{4}-3 j_{3}-3 j_{2}-3 j_{1}-8} \times U^{2} \\
& =U^{2} \sum_{a=m}^{\infty} \sum_{b=m}^{\infty} 2^{-3 j_{2}-3 j_{1}-4} \sum_{c=m}^{\infty} \sum_{d=m}^{\infty} 2^{-3 j_{4}-3 j_{3}-4},
\end{aligned}
$$

therefore we can derive

$$
\left\|e_{a, b, c, d}\right\|^{2} \leq U^{2} \times \frac{1}{9 m^{4}} \times \frac{1}{9 m^{4}}=\frac{U^{2}}{81 m^{8}} .
$$

In other words

$$
\left\|e_{a, b, c, d}\right\| \leq \frac{U}{9 m^{4}}
$$

Theorem 4 If $g(s, t)$ be the exact solution of Eq. (1) and $\hat{g}_{m}(s, t)$ be HWFs approximate solution of (1) that is obtained by (9) and $U_{i}$ 's be defined in Theorem 3, then by using hypothesises

1. $\|g\| \leq A, \quad(s, t) \in[0,1) \times[0,1)$,

2. $\left\|K_{i}\right\| \leq Q_{i}, i=1,2,(x, y, s, t) \in[0,1) \times[0,1) \times[0,1) \times[0,1)$,

3. $O(x)=\sup _{x \in[0,1)}|B(x)|$,

4. $\left(Q_{1}+\frac{U_{1}}{9 m^{4}}+\left(Q_{2}+\frac{U_{2}}{9 m^{4}}\right) \times O(x) \times O(y)\right)<1$,

we have

$$
\left\|g-\hat{g}_{m}\right\| \leq \frac{\frac{M}{3 m^{2}}+\frac{U_{1} A}{9 m^{4}}+\frac{U_{2} A}{9 m^{4}} \times O(x) \times O(y)}{1-\left[Q_{1}+\frac{U_{1}}{9 m^{4}}+\left(Q_{2}+\frac{U_{2}}{9 m^{4}}\right) \times O(x) \times O(y)\right]} .
$$

Proof We can obtain

$$
\begin{aligned}
g(x, y)-\hat{g}_{m}(x, y) & \\
= & f(x, y)-\hat{f}_{m}(x, y) \\
& +\int_{0}^{y} \int_{0}^{x}\left(K_{1}(x, y, s, t) g(s, t)-\hat{K}_{1, m}(x, y, s, t) \hat{g}_{m}(s, t)\right) d s d t \\
& +\int_{0}^{y} \int_{0}^{x}\left(K_{2}(x, y, s, t) g(s, t)-\hat{K}_{2, m}(x, y, s, t) \hat{g}_{m}(s, t)\right) d B(s) d B(t) .
\end{aligned}
$$

The mean value theorem conclude

$$
\begin{aligned}
\left\|g-\hat{g}_{m}\right\| \leq & \left\|f-\hat{f}_{m}\right\|+x y\left\|K_{1} g-\hat{K}_{1, m} \hat{g}_{m}\right\| \\
& +B(x) B(y)\left\|K_{2} g-\hat{K}_{2, m} \hat{g}_{m}\right\| .
\end{aligned}
$$

By using two first hypothesis and Theorem 3 we obtain

$$
\begin{aligned}
\left\|K_{1} g-\hat{K}_{1, m} \hat{g}_{m}\right\| & \leq\left\|K_{1}\right\|\left\|g-\hat{g}_{m}\right\|+\left\|K_{1}-\hat{K}_{1, m}\right\|\left(\left\|g-\hat{g}_{m}\right\|+\|g\|\right) \\
& \leq Q_{1}\left\|g-\hat{g}_{m}\right\|+\frac{U_{1}}{9 m^{4}}\left(\left\|g-\hat{g}_{m}\right\|+A\right)
\end{aligned}
$$




$$
=\left(Q_{1}+\frac{U_{1}}{9 m^{4}}\right)\left\|g-\hat{g}_{m}\right\|+\frac{U_{1}}{9 m^{4}} A .
$$

Similarly for the stochastic case we get

$$
\begin{aligned}
\left\|K_{2} g-\hat{K}_{2, m} \hat{g}_{m}\right\| & \leq\left\|K_{2}\right\|\left\|g-\hat{g}_{m}\right\|+\left\|K_{2}-\hat{K}_{2, m}\right\|\left(\left\|g-\hat{g}_{m}\right\|+\|g\|\right) \\
& =\left(Q_{2}+\frac{U_{2}}{9 m^{4}}\right)\left\|g-\hat{g}_{m}\right\|+\frac{U_{2}}{9 m^{4}} A .
\end{aligned}
$$

By Theorem 2 and substituting Eqs. (12) and (13) in (14), we have

$$
\begin{aligned}
\left\|g-\hat{g}_{m}\right\| \leq & \frac{M}{3 m^{2}}+x y\left[\left(Q_{1}+\frac{U_{1}}{9 m^{4}}\right)\left\|g-\hat{g}_{m}\right\|+\frac{U_{1}}{9 m^{4}} A\right] \\
& +B(x) B(y)\left[\left(Q_{2}+\frac{U_{2}}{9 m^{4}}\right)\left\|g-\hat{g}_{m}\right\|+\frac{U_{2}}{9 m^{4}} A\right] .
\end{aligned}
$$

By taking sup we have

$$
\begin{aligned}
\| g & -\hat{g}_{m} \| \\
\leq & \frac{M}{3 m^{2}}+\sup _{x \in[0,1)} x \times \sup _{y \in[0,1)} y\left[\left(Q_{1}+\frac{U_{1}}{9 m^{4}}\right) \sup _{s \leq x, t \leq y}\left\|g-\hat{g}_{m}\right\|+\frac{U_{1}}{9 m^{4}} A\right] \\
& +\sup _{x \in[0,1)}|B(x)| \times \sup _{y \in[0,1)}|B(y)|\left[\left(Q_{2}+\frac{U_{2}}{9 m^{4}}\right) \sup _{s \leq x, t \leq y}\left\|g-\hat{g}_{m}\right\|+\frac{U_{2}}{9 m^{4}} A\right],
\end{aligned}
$$

so

$$
\left\|g-\hat{g}_{m}\right\| \leq \frac{\frac{M}{3 m^{2}}+\frac{U_{1} A}{9 m^{4}}+\frac{U_{2} A}{9 m^{4}} \times O(x) \times O(y)}{1-\left[Q_{1}+\frac{U_{1}}{9 m^{4}}+\left(Q_{2}+\frac{U_{2}}{9 m^{4}}\right) \times O(x) \times O(y)\right]},
$$

finally Theorem 3 of [11] complete the proof.

\section{Numerical Example}

To illustrate the effectiveness of the proposed method, are carried out three numerical examples in this section as the solution mean, the error mean, \%95 confidence interval and the length of the confidence interval are shown by $\bar{g}(x, y), \bar{e}(x, y), C I$ and $L C I$, respectively in Tables 1, 2 and 3. According to these tables by applying HWFs method, as $J$ increases, $\bar{e}(x, y)$ and LCI decrease, nearly. Also the results of this method are compared with the BPFs method $[10,11]$ and are displayed in Table 4. According to Table 4 we can say that HWFs mehod is better than the BPFs method at some points and also there are some other points that the BPFs method is an able method in those. In addition, $3 D$ graphs of the these examples are shown in Figs. 1, 2 and 3. Consider the following 2D-linear stochastic Volterra integral equations of the second kind:

\section{Example 1}

$g(x, y)=f(x, y)+\int_{0}^{y} \int_{0}^{x} x y s t g(s, t) d s d t+\int_{0}^{y} \int_{0}^{x}(x+y+s+t) g(s, t) d B(s) d B(t)$, 
Table 1 The numerical results to Example 1

\begin{tabular}{lllllll}
\hline$J$ & $2 M$ & $(x, y)$ & $\bar{g}(x, y)$ & $\bar{e}(x, y)$ & $(L, U)$ & $L C I$ \\
\hline 1 & 2 & $(0.12,0.37)$ & 0.9136 & 0.52231 & $(0.82015,2.12111)$ & 1.30096 \\
& & $(0.62,0.87)$ & 1.0256 & 0.61874 & $(0.90361,2.52031)$ & 1.61670 \\
2 & \multirow{2}{*}{8} & $(0.19,0.31)$ & 0.8564 & 0.58270 & $(0.36914,1.50694)$ & 1.13780 \\
& \multirow{2}{*}{16} & $(0.69,0.94)$ & 0.6846 & 0.49231 & $(0.53103,1.14062)$ & 0.60959 \\
& & $(0.16,0.34)$ & 1.6913 & 0.21044 & $(1.54109,1.90764)$ & 0.36655 \\
& \multirow{2}{*}{32} & $(0.53,0.91)$ & 0.7318 & 0.17455 & $(0.52810,0.85127)$ & 0.32317 \\
& & $(0.30,0.76)$ & 0.8456 & 0.22661 & $(0.81154,1.20124)$ & 0.38970 \\
& & $(0.58,0.92)$ & 0.5962 & 0.09197 & $(0.54120,0.71061)$ & 0.16941 \\
\hline
\end{tabular}

Table 2 The numerical results to Example 2

\begin{tabular}{lllllll}
\hline$J$ & $2 M$ & $(x, y)$ & $\bar{g}(x, y)$ & $\bar{e}(x, y)$ & $(L, U)$ & $L C I$ \\
\hline 1 & 2 & $(0.12,0.37)$ & 0.5201 & 0.62163 & $(0.48156,1.85401)$ & 1.37245 \\
& & $(0.62,0.87)$ & 0.4545 & 0.34438 & $(0.41086,1.71244)$ & 1.30158 \\
2 & 8 & $(0.19,0.31)$ & 0.4891 & 0.44962 & $(0.25116,1.34201)$ & 1.09085 \\
& \multirow{2}{*}{16} & $(0.69,0.94)$ & 0.5709 & 0.45031 & $(0.22164,1.31132)$ & 1.08963 \\
3 & & $(0.16,0.34)$ & 0.5228 & 0.16420 & $(0.48511,0.92120)$ & 0.43609 \\
& \multirow{2}{*}{32} & $(0.53,0.91)$ & 0.6814 & 0.18010 & $(0.43160,0.87492)$ & 0.44332 \\
4 & $(0.30,0.76)$ & 0.7001 & 0.08460 & $(0.65320,0.89418)$ & 0.24098 \\
& & $(0.58,0.92)$ & 0.4667 & 0.11020 & $(0.42293,0.69409)$ & 0.27116 \\
\hline
\end{tabular}

Table 3 The numerical results to Example 3

\begin{tabular}{lllllll}
\hline$J$ & $2 M$ & $(x, y)$ & $\bar{g}(x, y)$ & $\bar{e}(x, y)$ & $(L, U)$ & $L C I$ \\
\hline 1 & 2 & $(0.12,0.37)$ & 0.6143 & 0.21438 & $(0.51617,0.91564)$ & 0.39947 \\
& & $(0.62,0.87)$ & 0.2182 & 0.25460 & $(0.19851,0.75694)$ & 0.55843 \\
2 & \multirow{2}{*}{8} & $(0.19,0.31)$ & 0.5149 & 0.19460 & $(0.36180,0.50934)$ & 0.14754 \\
& \multirow{2}{*}{16} & $(0.69,0.94)$ & 0.3939 & 0.18281 & $(0.34108,0.58447)$ & 0.24339 \\
& & $(0.16,0.34)$ & 0.5196 & 0.19400 & $(0.48156,0.71601)$ & 0.23445 \\
& \multirow{2}{*}{3} & $(0.53,0.91)$ & 0.5994 & 0.13327 & $(0.48485,0.75462)$ & 0.26977 \\
& \multirow{2}{*}{32} & $(0.30,0.76)$ & 0.6996 & 0.14025 & $(0.66451,0.73124)$ & 0.06673 \\
& & $(0.58,0.92)$ & 0.4984 & 0.05612 & $(0.44640,0.52681)$ & 0.08041 \\
\hline
\end{tabular}

where

$$
\begin{aligned}
f(x, y)= & (x+y)\left[1-\frac{1}{6} x^{3} y^{3}-\left(x B(x)-\int_{0}^{x} B(s) d s\right) B(y)\right. \\
& \left.-\left(y B(y)-\int_{0}^{y} B(t) d t\right) B(x)\right]-\left(x^{2} B(x)-2 \int_{0}^{x} s B(s) d s\right) B(y) \\
& -2\left(x B(x)-\int_{0}^{x} B(s) d s\right)\left(y B(y)-\int_{0}^{y} B(t) d t\right)
\end{aligned}
$$


Table 4 Comparison of the numerical results for HWFs method with BPFs method for Examples 1, 2 and 3

\begin{tabular}{|c|c|c|c|c|c|}
\hline Example & $J$ & $(x, y)$ & Method & $\bar{e}(x, y)$ & $L C I$ \\
\hline \multirow[t]{6}{*}{1} & \multirow[t]{2}{*}{1} & \multirow[t]{2}{*}{$(0.37,0.87)$} & BPFs & 0.12101 & 1.23060 \\
\hline & & & HWFs & 0.11569 & 0.85423 \\
\hline & \multirow[t]{2}{*}{3} & \multirow[t]{2}{*}{$(0.16,0.91)$} & BBFs & 0.01892 & 0.85446 \\
\hline & & & HWFs & 0.21520 & 0.23148 \\
\hline & \multirow[t]{2}{*}{4} & \multirow[t]{2}{*}{$(0.64,0.89)$} & BBFs & 0.20236 & 0.75129 \\
\hline & & & HWFs & 0.13740 & 0.81453 \\
\hline \multirow[t]{6}{*}{2} & \multirow[t]{2}{*}{1} & \multirow[t]{2}{*}{$(0.37,0.87)$} & BPFs & 0.23001 & 0.46501 \\
\hline & & & HWFs & 0.01475 & 0.15047 \\
\hline & \multirow[t]{2}{*}{3} & \multirow[t]{2}{*}{$(0.16,0.91)$} & BBFs & 0.11254 & 0.42718 \\
\hline & & & HWFs & 0.23160 & 0.08297 \\
\hline & \multirow[t]{2}{*}{4} & \multirow[t]{2}{*}{$(0.64,0.89)$} & BBFs & 0.04156 & 0.13451 \\
\hline & & & HWFs & 0.01266 & 0.02828 \\
\hline \multirow[t]{6}{*}{3} & \multirow[t]{2}{*}{1} & \multirow[t]{2}{*}{$(0.37,0.87)$} & BPFs & 0.19007 & 0.25425 \\
\hline & & & HWFs & 0.10105 & 0.36061 \\
\hline & \multirow[t]{2}{*}{3} & \multirow[t]{2}{*}{$(0.16,0.91)$} & $\mathrm{BBFs}$ & 0.00892 & 0.56490 \\
\hline & & & HWFs & 0.05141 & 0.54001 \\
\hline & \multirow[t]{2}{*}{4} & \multirow[t]{2}{*}{$(0.64,0.89)$} & BBFs & 0.15230 & 0.31640 \\
\hline & & & HWFs & 0.05857 & 0.03114 \\
\hline
\end{tabular}
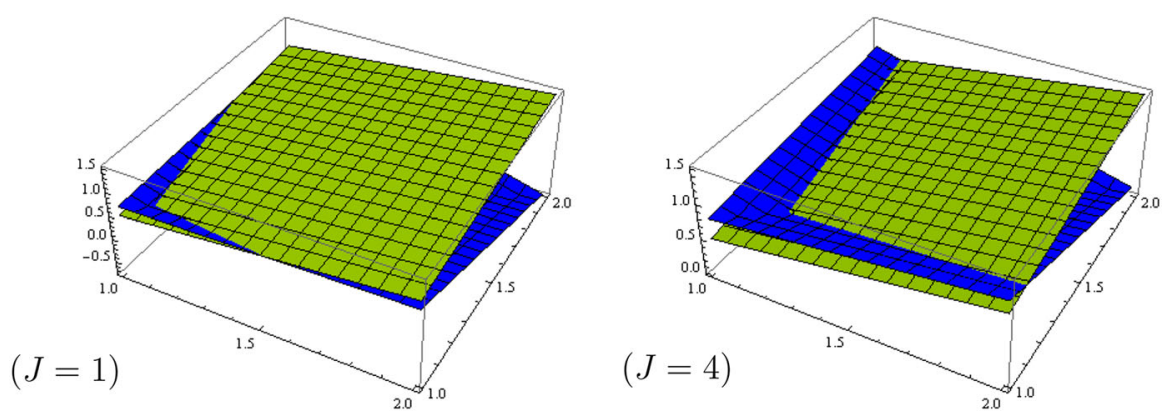

Fig. 1 Solutions of Example 1
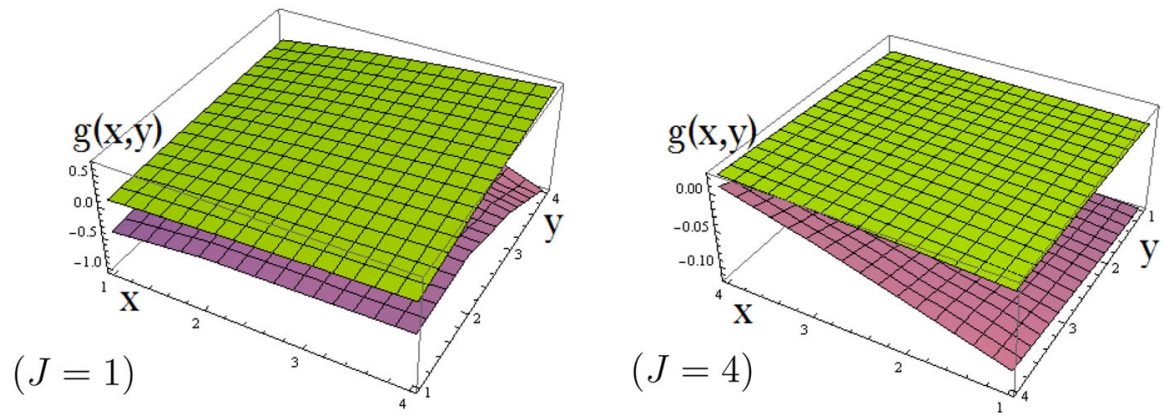

Fig. 2 Solutions of Example 2 

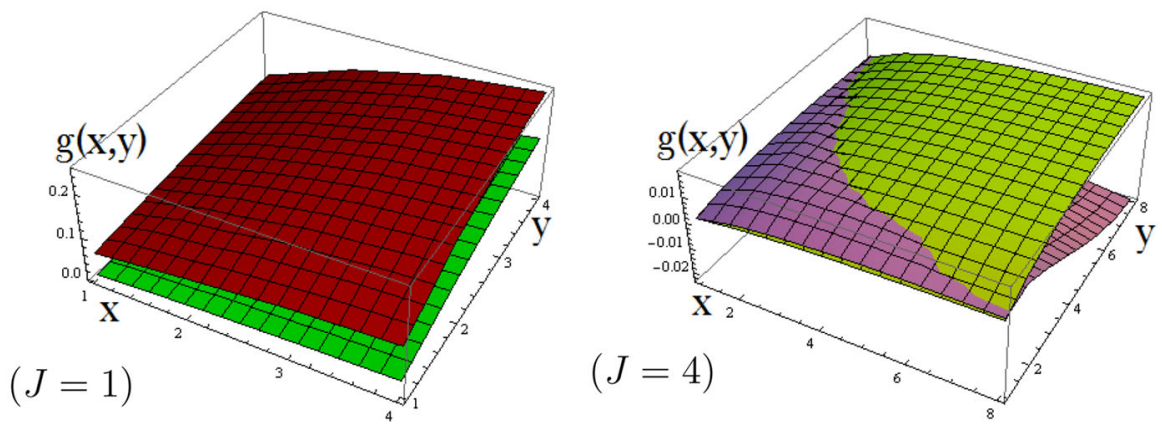

Fig. 3 Solutions of Example 3

$$
-\left(y^{2} B(y)-2 \int_{0}^{y} t B(t) d t\right) B(x),
$$

with the exact solution $g(x, y)=x+y$.

\section{Example 2}

$g(x, y)=f(x, y)+\int_{0}^{y} \int_{0}^{x}(x+y+s+t) g(s, t) d s d t+\int_{0}^{y} \int_{0}^{x} x y s t g(s, t) d B(s) d B(t)$,

where

$$
\begin{aligned}
f(x, y)= & B(x) B(y)-(x+y) \int_{0}^{y} B(s) d s \int_{0}^{x} B(s) d s-y \int_{0}^{x} s B(s) d s \\
& -x \int_{0}^{y} s B(s) d s-x y\left(\frac{y}{2} B^{2}(y)-\frac{y^{2}}{4}-\frac{1}{2} \int_{0}^{y} B^{2}(s) d s\right) \\
& \times\left(\frac{x}{2} B^{2}(x)-\frac{x^{2}}{4}-\frac{1}{2} \int_{0}^{x} B^{2}(s) d s\right),
\end{aligned}
$$

by the exact solution $g(x, y)=B(x) B(y)$.

\section{Example 3}

$$
\begin{aligned}
g(x, y)= & f(x, y)+\int_{0}^{y} \int_{0}^{x} t \sqrt{x+y} \operatorname{Sin}(s) g(s, t) d s d t \\
& +\int_{0}^{y} \int_{0}^{x} \operatorname{st} \operatorname{Cos}(x y) g(s, t) d B(s) d B(t),
\end{aligned}
$$

where

$$
\begin{aligned}
f(x, y)= & (x y) e^{B(x)+B(y)}-\int_{0}^{y} \int_{0}^{x} t^{2} s \sqrt{x+y} \operatorname{Sin}(s) e^{B(s)+B(t)} d s d t \\
& \left.-\operatorname{Cos}(x y)\left(x e^{B(x)}-\int_{0}^{x} e^{B(s)}\left(1+\frac{s}{2}\right) d s\right)\right) \\
& \left.\left(y e^{B(y)}-\int_{0}^{y} e^{B(s)}\left(1+\frac{s}{2}\right) d s\right)\right),
\end{aligned}
$$

by the exact solution $g(x, y)=(x y) e^{B(x)+B(y)}$. 


\section{Conclusion}

In this paper, we developed HWFs numerical method for approximate a solution of Eq. (1). The error analysis and the numerical examples show accuracy of this method. Furthermore, the current method can be run with increasing $J$ until the results settle down to an appropriate accuracy that it leads to solving $2^{2 J+2}$ linear systems, that have its difficulties. Finally, this method can be extended and applied to $2 \mathrm{D}$ linear or non-linear multi-noise stochastic VolterraFredholm integral equations of the first or second kind.

Open Access This article is distributed under the terms of the Creative Commons Attribution 4.0 International License (http://creativecommons.org/licenses/by/4.0/), which permits unrestricted use, distribution, and reproduction in any medium, provided you give appropriate credit to the original author(s) and the source, provide a link to the Creative Commons license, and indicate if changes were made.

\section{References}

1. Atkinson, K.E.: The Numerical Solution of Integral Equations of the Second Kind. Cambridge University Press, New York (1997)

2. Jerri, A.J.: Introduction to Integral Equations with Applications. Wiley, New York (1999)

3. Aziz, I., Siraj-ul, I., Khan, F.: A new method based on Haar wavelet for numerical solution of twodimensional nonlinear integral equations. J. Comput. Appl. Math. (2014). https://doi.org/10.1016/j.cam. 2014.04.027

4. Guoqiang, H., Jiong, W.: Extrapolation of nystrom solution for two dimentional nonlinear Fredholm integral equations. J. Comput. Appl. Math. 134, 259-268 (2001)

5. Guoqiang, H., Itayami, K., Sugihara, K., Jiong, W.: Extrapolation method of iterated collocation solution for two-dimentional nonlinear Volterra integral equations. Appl. Math. Comput. 112, 49-61 (2000)

6. Xie, W., Lin, F.R.: A fast numerical solution method for two dimensional Fredholm integral equations of the second kind. Appl. Numer. Math. 59, 1709-1719 (2009)

7. Maleknejad, K., JafariBehbahani, Z.: Application of two-dimensional triangular functions for solving nonlinear class of mixed Volterra-Fredholm integral equations. Math. Comput. Model. 55, 1833-1844 (2012)

8. Babolian, E., Maleknejad, K., Roodaki, M., Almasieh, H.: Two dimensional triangular functions and their applications to nonlinear 2D Volterra-Fredholm equations. Comput. Math. Appl. 60, 1711-1722 (2010)

9. Fallahpour, M., Khodabin, M., Maleknejad, K.: Approximation solution of two-dimensional linear stochastic fredholm integral equation by applying the Haar wavelet. Math. Model. Comput. 5, 361-372 (2015)

10. Fallahpour, M., Khodabin, M., Maleknejad, K.: Approximation solution of two-dimensional linear stochastic Volterra-Fredholm integral equation via two-dimensional block-pulse functions. Int. J. Ind. Math. 8(4), IJIM-00774 (2016)

11. Fallahpour, M., Khodabin, M., Maleknejad, K.: Theoretical error analysis and validation in numerical solution of two-dimensional linear stochastic Volterra-Fredholm integral equation by applying the blockpulse functions. Cogent Math. 4, 1296750 (2017)

12. Fallahpour, M., Khodabin, M., Ezzati, R.: A new computational method based on Bernstein operational matrices for solving two-dimensional Linear stochastic Volterra integral equations. Differ. Equ. Dyn. Syst. (2019). https://doi.org/10.1007/s12591-019-00474-y

13. Jiang, G., Sang, X., Wu, J., Li, B.: Numerical solution of two-dimensional nonlinear stochastic Itô-Volterra integral equations by applying block-pulse functions. Adv. Pure Math. 9, 53-66 (2019)

14. Mirzaee, F., Samadyar, N.: Using radial basis functions to solve two dimensional linear stochastic integral equations on non-rectangular domains. Eng. Anal. Boundary Elem. 000, 1-16 (2018)

15. Rudin, W.: Principles of Mathematical Analysis. McGraw-Hill, New York (1976)

16. Mohammadi, F.: Haar wavelets approach for solving multidimensional stochastic Itó-Volterra integral equations. Appl. Math. E-Notes 15, 80-96 (2015)

Publisher's Note Springer Nature remains neutral with regard to jurisdictional claims in published maps and institutional affiliations. 